\title{
Grain selection and growth orientation of prior- $\beta$ phase for Ti-6-4 during additive manufacturing: insights from a modeling perspective
}

\author{
Wei-zhao Sun', Fei-hu Shan', Nan-fu Zong', * *Hong-biao Dong ${ }^{3}$, 'Tao Jing' \\ 1. Key Laboratory for Advanced Materials Processing Technology, Ministry of Education, School of Materials Science and Engineering, \\ Tsinghua University, Beijing 100084, China \\ 2. Science and Technology on Power Beam Processes Laboratory, AVIC Beijing Aeronautical Manufacturing Technology Research Institute, \\ Beijing 100024, China \\ 3. School of Engineering, University of Leicester, LE1 7RH, UK
}

\begin{abstract}
The microstructure of Ti-6-4 components produced by additive manufacturing suffers from the coarse and elongated prior- $\beta$ grain, which leads to a decrease of the tensile behavior and the occurrence of anisotropy. To understand and control the grain evolution, a multiscale simulation is applied to investigate the relationship between the grain selection, growth orientation, and the molten pool morphology with the different deposition layer numbers and processing parameters. The accuracy of the simulation is validated by experiments in both qualitative and quantitative ways. Results show that when the grain with unfavorable orientation loses the competitive growth with its neighbors, there will be a great chance that the blocked grain is eliminated in the following layer-and-layer deposition, which leads to the increase of the grain width. The size of the molten pool increases remarkably as the layer number increases, which lays a heavy burden on the stability of the molten pool. The analytical relationship between the molten pool morphology and the grain growth orientation is also deduced. The flat molten pool causes the grains with the $<001>$ direction close to the building direction to have greater survival potential. Besides, decreasing the line power energy shows little effect on the stability of the molten pool and the grain growth orientation, especially when the deposited layer number is large. The revealing mechanisms will help in understanding and further controlling the grain evolution.
\end{abstract}

Key words: additive manufacturing; Ti-6-4; simulation; grain selection; grain growth

CLC numbers: TG146.23 Document code: A

Article ID: 1672-6421(2021)02-083-11

\section{Introduction}

Ti-6-4 is one of the most widely used industrial alloys in additive manufacturing (AM). Different from other alloys used in AM, the columnar-to-equiaxed transition (CET) of Ti-6-4 is rather challenging and not likely to occur in the general AM process ${ }^{[1]}$. With the high localized heat input, the additively manufactured (AMed) Ti-6-4 microstructure is usually characterized by the coarse and elongated $\beta$ grain, which leads to the

\footnotetext{
*Tao Jing
}

Male, born in 1965, Professor. His research interests mainly focus on materials processing technology and integrated computational material engineering (ICME). To date, he has published more than 100 papers.

E-mail: jingtao@mail.tsinghua.edu.cn;

**Hong-biao Dong

E-mail: h.dong@le.ac.uk;

Received: 2020-11-10; Accepted: 2021-02-26 decrease of the tensile behavior ${ }^{[2]}$ and the occurrence of anisotropy ${ }^{[3]}$. To find out what exactly the grains experience will help in understanding the grain evolution and further controlling it.

Pioneering results ${ }^{[4,5]}$ report to understand the elongated $\beta$ grain evolution by ex-situ characterizations. For example, Thijs et al. ${ }^{[4]}$ comprehensively studied the influence of the scanning parameters and the scanning strategy on the microstructure of Ti-6-4 during selective laser melting. Antonysamy et al. ${ }^{[5]}$ investigated the effects of geometry on the prior- $\beta$ grain structure and texture and found that the $<001>$ direction tended along the building direction. For the lack of in-situ characterizations, the above type of research adopted the assumption that the grain direction is parallel with the local conductive heat transfer. And hereby the grain orientation by thermal transfer simulation is inferred and explained. However, there exists inconsistency between the assumption and the solidification theory. 
As shown in Fig. 1, from the bottom to the top of the rear part of molten pool, the direction of the temperature gradient ( $\mathrm{G}$, the red arrow in Fig. 1) varies with position. Based on the classical welding theory, the favorable orientations of the grains at different positions are different as well. Thus, it is not predictable which favorable orientation will survive in the end, i.e., whether the grains with $<001>$ direction (the green arrow in Fig. 1) along the building direction will survive or not cannot be determined. Suppose that we agree that the grains with $<001>$ direction will win the competitive growth at last, however, during the growth at the solid-liquid (S-L) interface, the $<001>$ direction is not along the local temperature gradient for most cases. The studies about solidification ${ }^{[6-8]}$ suggest that when the grain direction is inconsistent with its local temperature gradient, the grain will grow in an intermediate direction. Therefore, the assumption needs to be corrected and improved that the grain growth direction is along the temperature gradient.

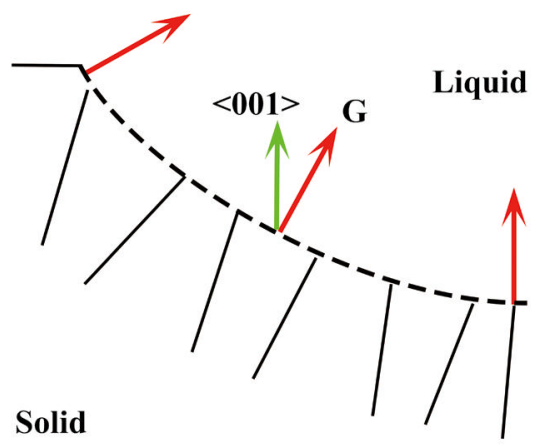

Fig. 1: Schematic of grain evolution during AM (grain boundary and molten pool edge are distinguished by solid and dashed line)

With the blooming development of solidification modeling ${ }^{[9-12]}$, solidification microstructure modeling provides an available way to investigate the in-situ grain evolution. Currently, cellular automaton (CA) ${ }^{[9,12,13]}$ and phase-field (PF) method ${ }^{[11,14]}$ are the two main solidification simulation methods. Although PF method shows advantages on the dendrite simulation with detailed information, such as the primary, secondary dendrite and the concentration distribution, it is also notorious for its enormous computational cost. Therefore, it is often used to simulate single or several grains with the simulation box of several hundred micrometers. In contrast, the CA method ignores the details of the dendrite and tracks the envelope of the dendrite. Therefore, it has a much lower computational cost and can be used to simulate hundreds or thousands of grains, in which the simulation box is at the scale of several millimeters.

Pioneering research works ${ }^{[9,12,15,16]}$ introduced the CA method into AM modeling. Gandin and his co-workers ${ }^{[9,15]}$ have developed a multiscale framework to couple the thermal transfer with grain evolution by the CA method. In the framework, they firstly calculated the thermal transfer with the coarse grid and then embedded it into the fine CA grid. The framework is followed by researchers ${ }^{[12,17,18]}$ and shows promising prospects in the solidification modeling during AM. The framework can be improved by focusing on the grain coarsening in the heataffected zone (HAZ) ${ }^{[19]}$ and correcting the dendrite kinetics ${ }^{[14]}$. Another focus is that the grains of AM or welding grow in an epitaxial way and are affected by the grain size of the substrate. Wei et al. ${ }^{[19]}$ used the Monte Carlo (MC) method to simulate grain coarsening at the HAZ and to initialize the grain size for epitaxial growth. As for the dendrite kinetics, which is the growth velocity as a function of undercooling, our previous research ${ }^{[14]}$ suggests that it can be obtained from the PF method.

In conclusion, the research proposes to investigate grain evolution during AM by a multiscale microstructure simulation method. The relationship between the grain selection and the growth orientation is studied. The research is organized as follows: In Section 2, the theoretical part is briefly introduced. In Section 3, the validations of the simulation are discussed, and then the grain competitive growth and grain elimination are investigated by the in-situ grain evolution. Finally, the molten pool morphology, grain selection and orientation are investigated to answer the question as to why the texture with the $<001>$ orientation along the building direction is formed.

\section{Methods}

CA method essentially is a cell-capturing method, determining which and when the cell is captured. Originally, Rappaz and Gandin ${ }^{[16,20,21]}$ introduced the CA method to simulate the solidification microstructure during casting. They improved the accuracy of the model by the decentered-square capturing method, which is currently the most widely used method. Here, the decentered-square capturing method is briefly discussed. Details can be found in Ref. [21]. Currently, two-dimensional (2D) ${ }^{[22]}$ and three-dimensional (3D) ${ }^{[15]} \mathrm{CA}$ methods are well used. Although the $3 \mathrm{D}$ method shows the advantage on the accuracy of simulation, the efficient 2D method is adequate for this particular case. The reason is that the $\beta$ grain is relatively large in both the transversal and longitudinal planes and the competitive growth, what happens in $3 \mathrm{D}$ domain in reality, is reasonable to be simplified as a $2 \mathrm{D}$ case.

As shown in Fig. 2, suppose nucleation happens at the position of $v$. For the body-centered cubic $\beta$ phase, the nucleus will grow with the shape of the square. The half size of the square $L_{v_{t}}$ at $v$ position at time $t$ is

$$
L_{v_{t}}=\frac{1}{\sqrt{2}} \int_{0}^{t} v(\Delta T) d t
$$

where $v(\Delta T)$ is the primary growth velocity as a function of undercooling. When the size of the square is large enough, the nodes around it will be captured. Taking the $\mu$ node as an example, the square at $\mu$ position will grow with a new square (the red square in Fig. 2). The initial half size of the square at $\mu$ position is

$$
L_{\mu_{t}}=\frac{1}{2}\left[\min \left(L_{\mu_{t}}^{[11]}, \sqrt{2} d x\right)+\min \left(L_{\mu_{t}}^{[\overline{1} \overline{1}]}, \sqrt{2} d x\right)\right]
$$


where $d x$ is the mesh size. The square at $\mu$ position shares the same dendrite tip with the square at $v$ position.

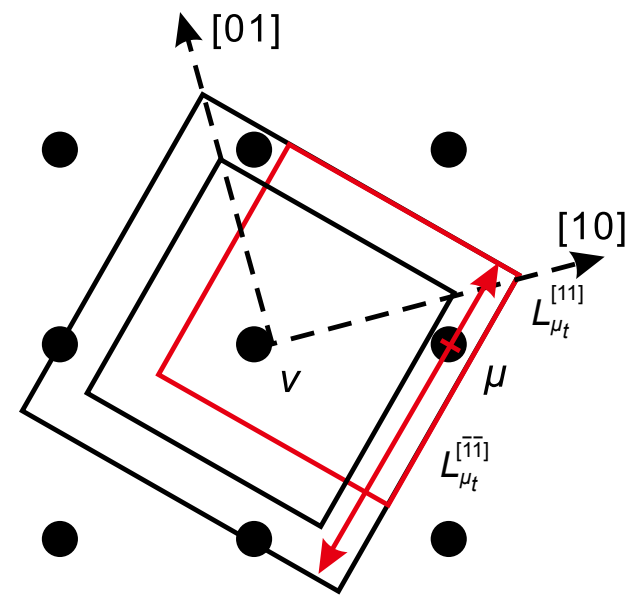

Fig. 2: Schematic diagram of decentered square capturing method

It should be noticed that it is not the details of dendrites but the envelopes of grains that are simulated by CA method. Our main concern here is not the details of the dendrite, so the CA method is a proper choice. According to Eq. (1), the growth of grains in CA is determined by both the dendrite kinetics $v(\Delta T)$ and the local temperature. The temperature can be obtained from thermal-transfer simulation, as it has been presented in Ref. [9]. Concerning the effects of grain growth on the thermal-transfer analysis, it is acceptable to calculate it firstly and then to embed the thermal distribution into CA method. As the mesh size for thermal simulation is far larger than that for CA method, it is usual practice that the temperature field calculated from the coarse mesh is interpolated into fine CA mesh to reduce computational cost. The Monte Carlo method is also applied to simulate the grain coarsening in the heataffected zone (HAZ). As it is not the main concern here, readers may refer to Refs. [19] and [23].

\section{Results and discussion}

\subsection{Validation}

Experiments are carried out by using a self-developed wire laser additive manufacturing (WLAM) machine developed by AVIC Manufacturing Technology Institute (China). Singlelayer and five-layer Ti-6-4 are deposited when the laser power is $2,500 \mathrm{~W}$ or $3,000 \mathrm{~W}$ onto a rolled substrate with the size of $100 \mathrm{~mm} \times 100 \mathrm{~mm} \times 10 \mathrm{~mm}$. The schematic diagram of the scanning strategy is shown in Fig. 3. The scanning velocity is $10 \mathrm{~mm} \cdot \mathrm{s}^{-1}$. The real laser radius is $3 \mathrm{~mm}$. The length of each deposition layer is $90 \mathrm{~mm}$. After each layer is deposited, the machine needs $3 \mathrm{~s}$ to be ready for the following deposition. Here, the microstructure with respect to the laser power and deposited layer was investigated because these two parameters are easy to be controlled and the processing window for WLAM is relatively narrow. Besides, the goal is not to obtain the most optimized processing parameter for experiments, but to validate the accuracy of the simulation model.

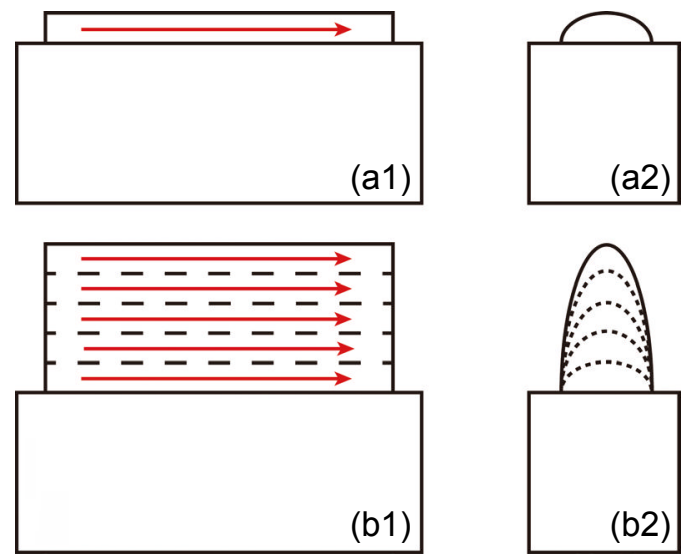

Fig. 3: Schematic diagram of scanning strategies from longitudinal [(a1) and (b1)] and transversal [(a2) and (b2)] views. One-layer deposited case: (a1) and (a2); Five-layer deposited case: (b1) and (b2)

Gaussian surface heat source ${ }^{[24]}$ was applied to simulate the thermal transfer, given by

$$
S=\frac{P d}{\pi r_{b}^{2}} \eta \exp \left(-d \frac{r^{2}}{r_{b}^{2}}\right)
$$

where $P, d=3, r_{b}, \eta$ and $r$ are the laser power, energy distribution factor, fitting laser radius, laser efficiency, and the distance between the laser power and the node, respectively. Material properties used for thermal transfer analysis can be found in Ref. [25].

The single-layer deposited case is used to fit the heat surface parameters. After fitting, for our cases, the fitting laser radius $r_{b}$ and efficiency $\eta$ are $7.5 \mathrm{~mm}$ and 0.45 . Results show that when the laser power is $2,500 \mathrm{~W}$, in the transversal plane, the molten pool width and depth are 5,081.324 $\mu \mathrm{m}$ and $694.952 \mu \mathrm{m}$ obtained from experiment, respectively, while they are 5,068.012 $\mu \mathrm{m}$ and $669.945 \mu \mathrm{m}$ obtained from simulation. The relative errors are $-0.3 \%$ and $-3.6 \%$. When the laser power is $3,000 \mathrm{~W}$, the molten pool width and depth are 5,607.690 $\mu \mathrm{m}$ and $966.148 \mu \mathrm{m}$ obtained from experiment, respectively, while they are 5,855.172 $\mu \mathrm{m}$ and $961.496 \mu \mathrm{m}$ obtained from simulation. The relative errors are $4.4 \%$ and $-0.5 \%$. The relative errors are less than $5.0 \%$. The processing modeling results agree with that obtained from experiments within limited errors.

The two-dimensional (2D) grain evolutions of the transversal (Figs. 4 and 5) and the central longitudinal (Figs. 6 and 7) planes are investigated. For the 2D simulation, the orientation is defined as the angle between $<01>$ direction of the grain with the building direction ${ }^{[21]}$. Take the anticlockwise direction as a positive direction, when the range of the initial angle is between $\pm \pi / 4$, the orientation of $2 \mathrm{D}$ grain is all covered.

The experimental and simulation results obtained from the transversal plane are shown in Figs. 4 and 5. As it can be seen, the two distinguished features involved in the AM, i.e., 
epitaxial and curved growth, have been calculated very well. The rolled substrate, the recrystallized and coarse $\beta$ grain featured HAZ and the coarse $\beta$ grain featured fusion zone (FZ) can be found from both the experiment and simulation. What is more, the three zones are very similar in experiment and simulation. Therefore, the simulation results are consistent with the experiment results from a qualitative perspective.
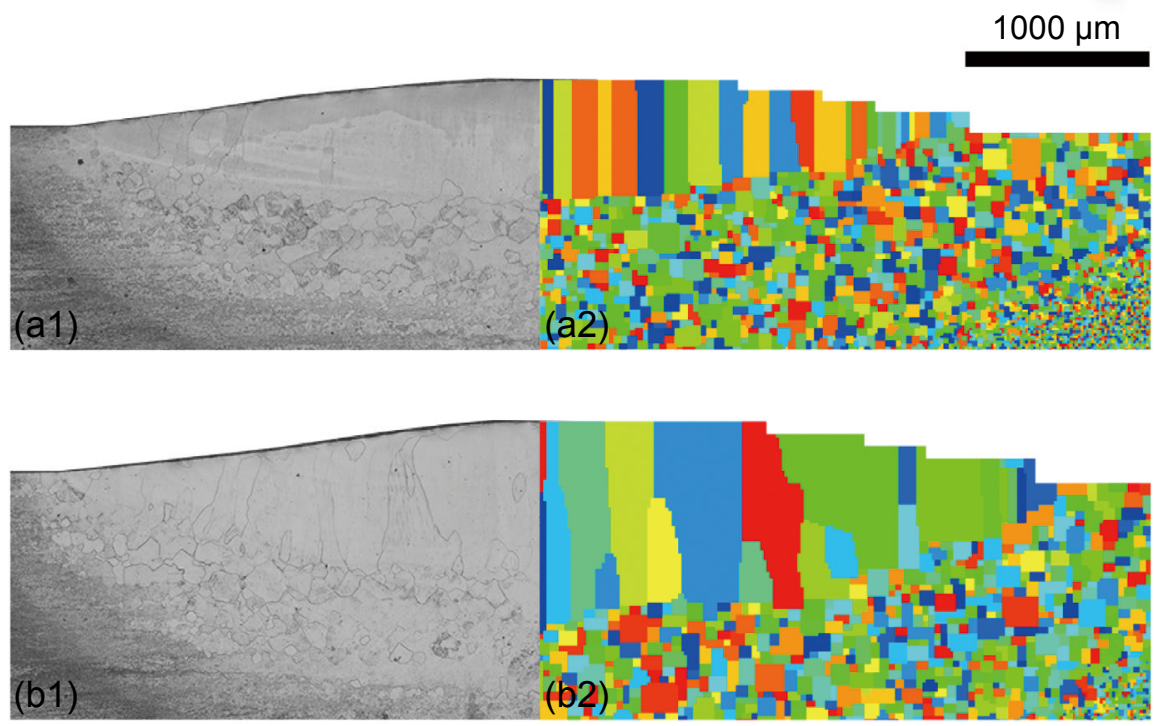

Fig. 4: Experimental (a1, b1) and simulation (a2, b2) results of transversal plane at different laser powers for single-layer deposited case: (a1), (a2) 2,500 W; (b1), (b2) 3,000 W

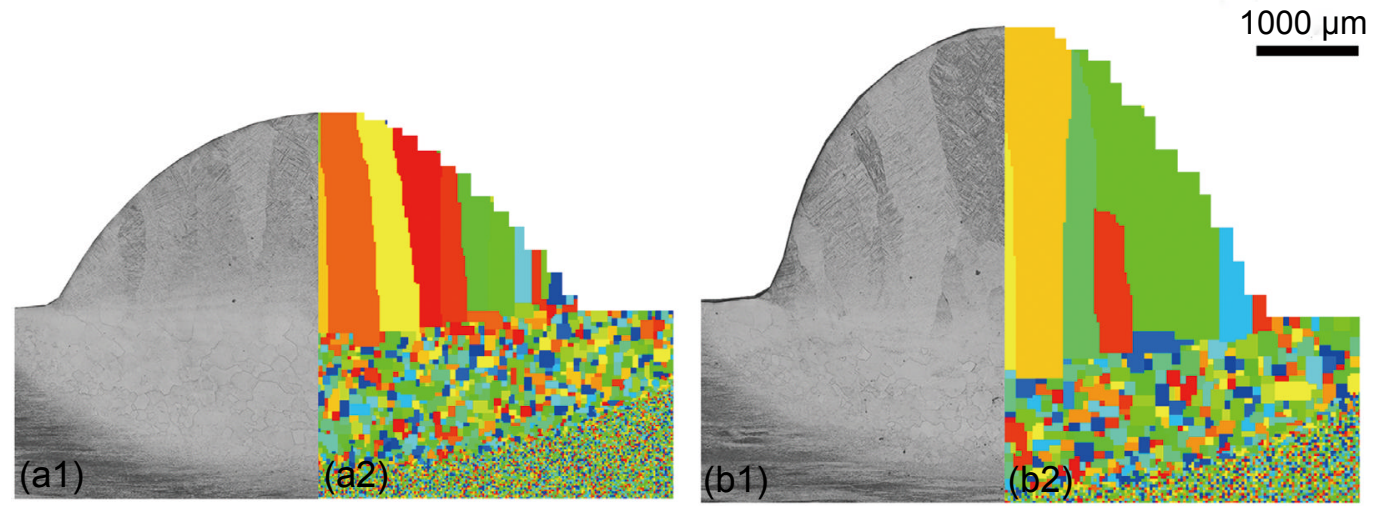

Fig. 5: Experimental (a1, b1) and simulation (a2, b2) results of transversal plane at different laser powers for five-layer deposited case: (a1), (a2) 2,500 W; (b1), (b2) 3,000 W

The microstructure in the longitudinal planes is also investigated. It is impossible to extract the central longitudinal plane of the experiment exactly. The microstructure of the longitudinal planes close to the central plane is illustrated in Figs. 6 and 7. To enhance visibility, the fusion line and the grain boundary are distinguished by the white and red dashed line in Figs. 6(a1) and (b1) and Figs. 7(a1) and (b1). The grains are distinguished by colors in Figs. 6(a2, b2) and Figs. 7(a2) and (b2). Taking the onelayer deposition case as an example, the depth of the molten pool in Figs. 6(a1) and (b1) is $640.244 \mu \mathrm{m}$ and $949.695 \mu \mathrm{m}$ obtained from experiment, which is similar with that from the central transversal plane $(694.952 \mu \mathrm{m}$ and $966.148 \mu \mathrm{m})$. Considering the grain is relatively large (several hundred micrometers in each direction), there is no significant difference between the central longitudinal plane and its neighboring longitudinal plane. Therefore, the microstructure in Figs. 6 and 7 can be roughly considered as that of the central longitudinal plane.
From the qualitative perspective, similar conclusions for the central longitudinal plane with that for transversal planes can be drawn. In the FZ, it can be found that as the laser power increases [Figs. 6(a2) and (b2), Figs. 7(a2) and (b2)], the grain grows more obliquely to the scanning direction. The trend agrees with what has been found in the experiment of Ref. [26]. The average grain width along the scanning direction is analyzed, which is defined as the average width along the scanning direction ${ }^{[2]}$. For the one-layer deposition case (Fig. 6), the grain width obtained from the simulation is $194.192 \mu \mathrm{m}$ and $307.317 \mu \mathrm{m}$ when the laser power is $2,500 \mathrm{~W}$ and $3,000 \mathrm{~W}$, respectively, while the grain width is $191.449 \mu \mathrm{m}$ and $309.263 \mu \mathrm{m}$ obtained from the experiment. The relative errors are $1.4 \%$ and $-0.6 \%$. For the fivelayer deposition case (Fig. 7), the grain width obtained from the simulation is $549.511 \mu \mathrm{m}$ and $824.267 \mu \mathrm{m}$ when the laser power is $2,500 \mathrm{~W}$ and $3,000 \mathrm{~W}$, respectively, and that the grain width is $521.368 \mu \mathrm{m}$ and $837.740 \mu \mathrm{m}$ obtained from the experiment. The 
relative errors are $-5.4 \%$ and $-1.6 \%$. Therefore, the accuracy of the model is at a relatively high level.

Although the grain actually grows in a three-dimensional domain, according to the microstructure of the transversal plane in Figs. 4 and 5, it can be found that the grain at the center of the molten pool is not blocked by the other grains and grows in the central longitudinal plane. In other words, the grains in the central longitudinal plane are not blocked by the grains in the other longitudinal planes. Therefore, when the competitive growth of the central longitudinal plane is investigated, it is reasonable to ignore the competitive growth in the transversal plane and reduce the $3 \mathrm{D}$ into $2 \mathrm{D}$ grain evolution case.
It is also noticed that there still exist discrepancies between simulations and experiments. For example, for the one-layer deposition case, it seems that the HAZ in the simulation is more oversized than that in the experiment. For the 3,000 W five-layer deposition case, if the average grain width is within the whole simulation box, the grain width will be $996.878 \mu \mathrm{m}$ instead of $837.740 \mu \mathrm{m}$. The disagreements may be attributed to the selected investigated region or to the simulation parameters. More work needs to be done to improve the simulation, however, it seems that the disagreements do not affect the objective of the research too much and hereby it is believed that the accuracy of the simulation is validated.
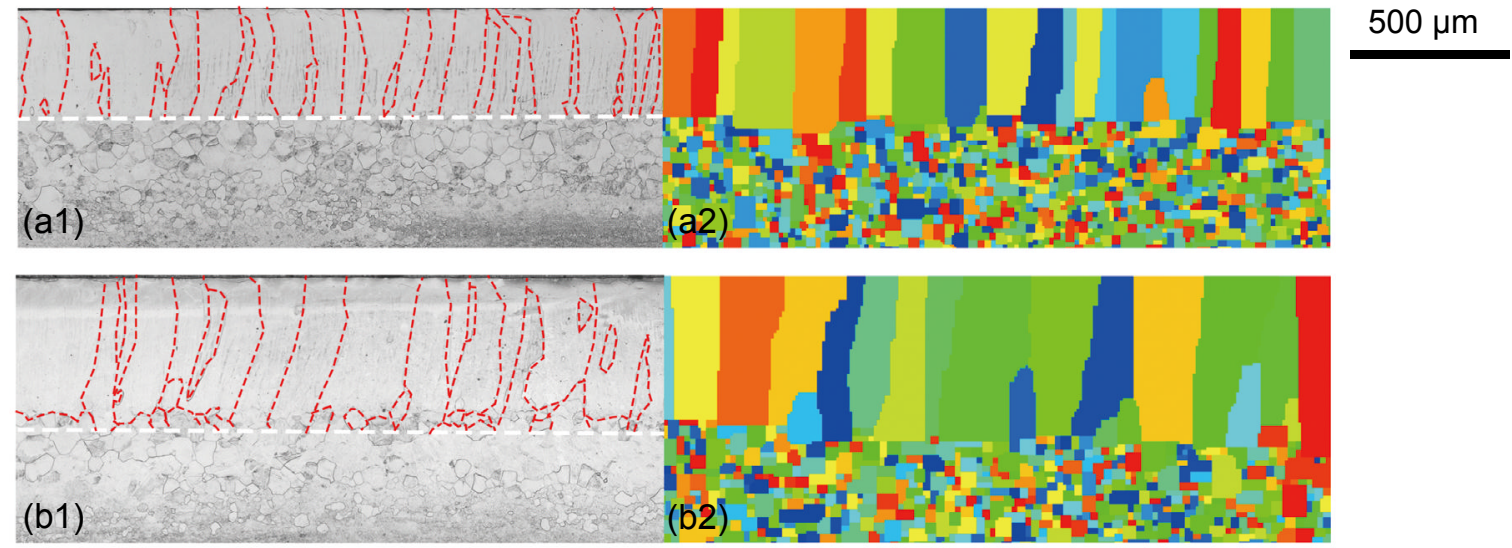

Fig. 6: Experimental (a1, b1) and simulation (a2, b2) results of longitudinal plane at different laser powers for one-layer deposited case: (a1), (a2) 2,500 W; (b1), (b2) 3,000 W
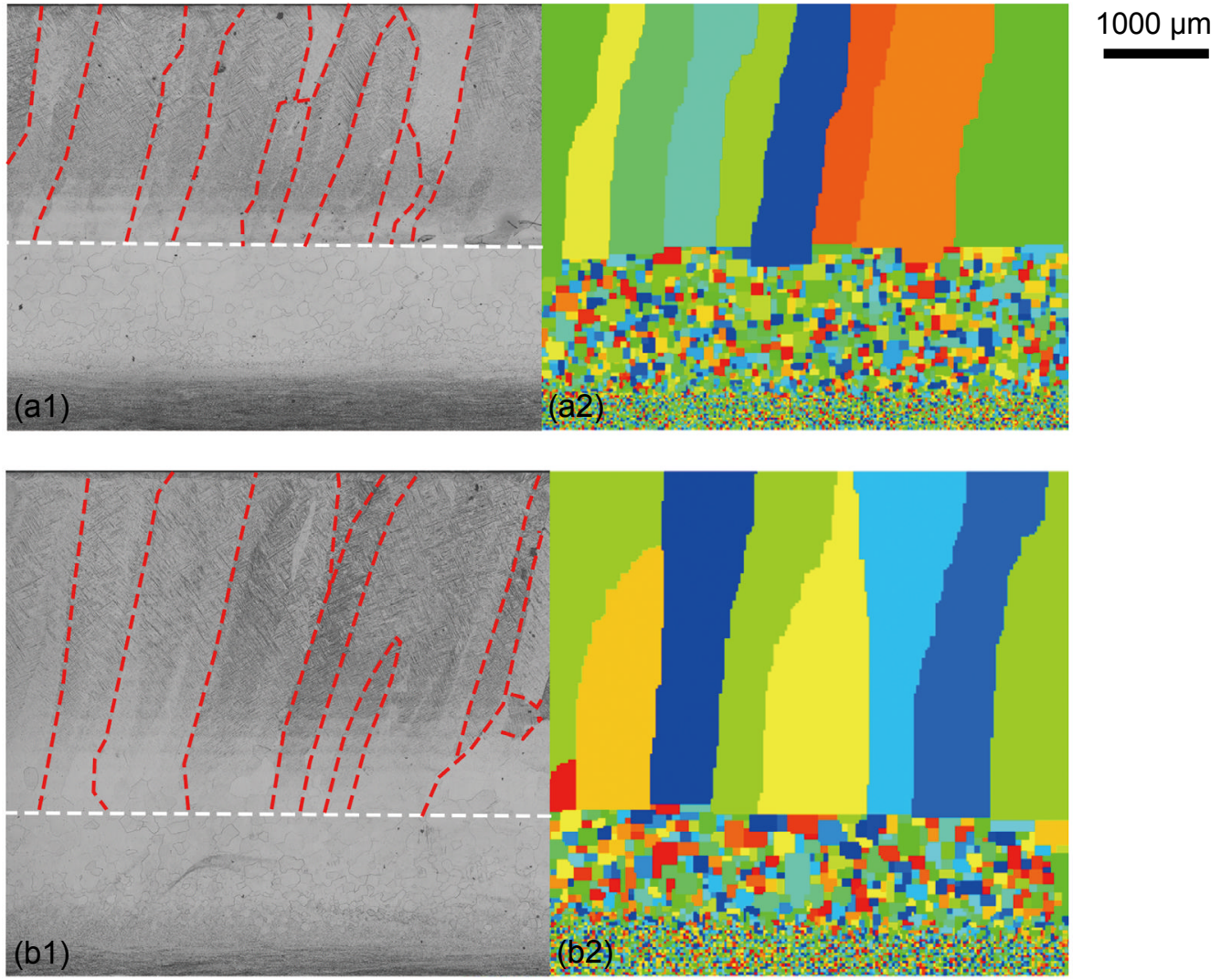

Fig. 7: Experimental (a1, b1) and simulation (a2, b2) results of longitudinal plane at different laser powers for five-layer deposited case: (a1), (a2) 2,500 W; (b1), (b2) 3,000 W 


\subsection{Grain width}

The relationship between the grain width and the deposited layer is studied. It can be found there is a significant increase in the grain width as the deposition layer increases. For the $3,000 \mathrm{~W}$ case, after the 1st and 5th layer deposition, the experimental grain width is $309.263 \mu \mathrm{m}$ and $837.740 \mu \mathrm{m}$. Ren et al. ${ }^{[2]}$ found that the mechanical performance of AMed Ti-6-4 products had a positive correlation with the grain width. Therefore, revealing the underlying mechanisms of the coarsening of the grain width helps in improving mechanical performance.

By investigating the grain evolution, it is found the coarsening of the grain width is attributed to the competitive growth and grain elimination. The competitive growth for the 3,000 W case is shown in Figs. 8(a1), (b1) and (c1). For the simplicity of expression, the chosen crystals [Fig. 8(a1)] are denoted as Crystals 1, 2, 3 and 4 with the orientation of $-0.544^{\circ}$, $41.301^{\circ}, 22.583^{\circ}$, and $5.304^{\circ}$, respectively. Figures 8(a1), (b1) and (c1) are extracted from the time step of $25.8 \mathrm{~s}, 26.1 \mathrm{~s}$, and $26.2 \mathrm{~s}$ during the $3 \mathrm{rd}$ layer deposition. Figures 8(a2), (b2) and (c2) are extracted from the time step of $37.2 \mathrm{~s}, 37.5 \mathrm{~s}$, and $38.0 \mathrm{~s}$ during the 4 th layer deposition. During the deposition of the 3rd layer, the three crystals begin to grow when the laser moves away and the temperature decreases [Fig. 8(a1)]. However, the orientation of Crystals 2 and 3 is unfavorable and they will fall behind the growth of neighbor Crystals 1 and 4 . The growth space for Crystals 2 and 3 is squeezed [Fig. 8(b1)] and they finally lose their competitive growth with Crystals 1 and 4 [Fig. 8(c1)].

During the following 4th deposition, before the laser moves right above Crystals 1, 2, 3, and 4, the temperature increases. When the temperature is above a certain level, the grain boundary has enough energy to move between Crystals 1 and 2, Crystals 2 and 3, and Crystals 3 and 4, when the four crystals are at the HAZ. Because the grain boundary moves randomly, which means it can be moved from Crystal 1 to Crystal 2 or moves inversely, the overall trend is to decrease the total energy, i.e., eliminate the grain boundary. Figures 8(a2) to (b2) show that Crystals 2 and 3 are pushed to the substrate and far below the molten pool finally. Therefore, when the laser moves away and the temperature decreases, Crystal 2 is not able to serve as an epitaxial growth site [Fig. 8(c2)]. After the layer-and-layer deposition, the grain with favorable orientation survives at last.
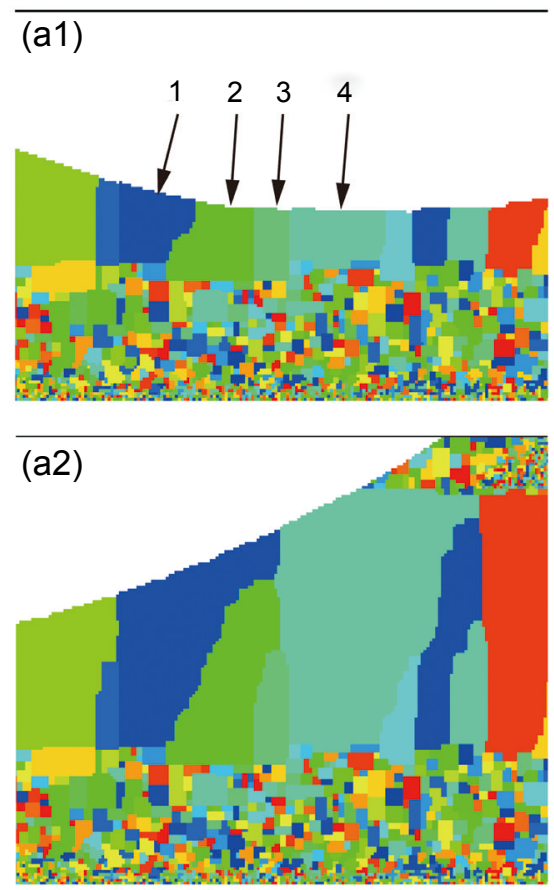

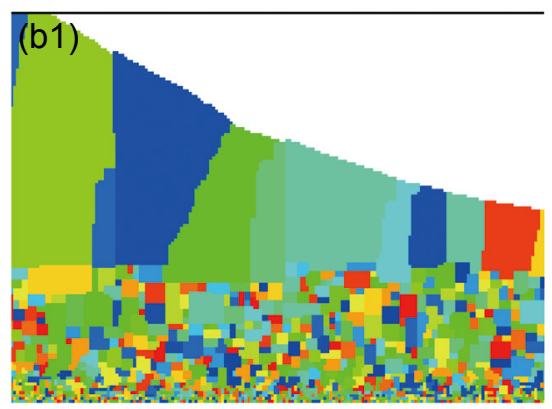

(b2)

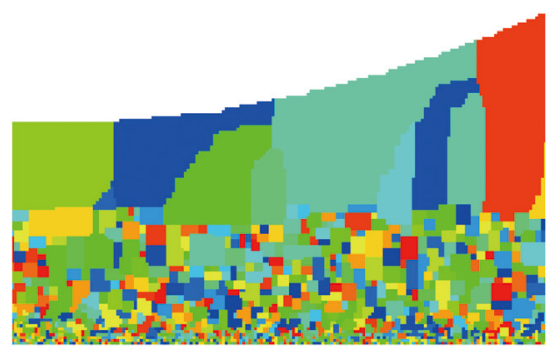

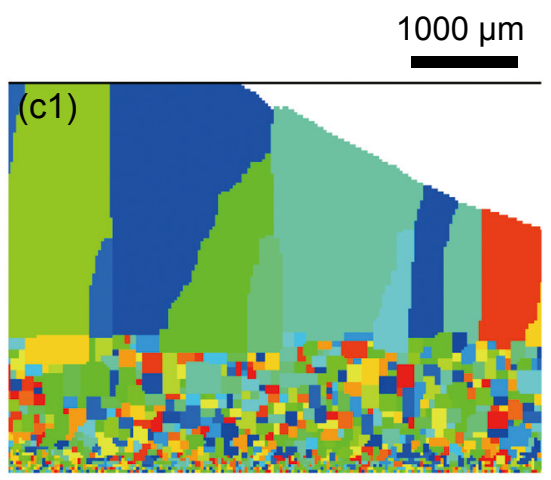

(c2)

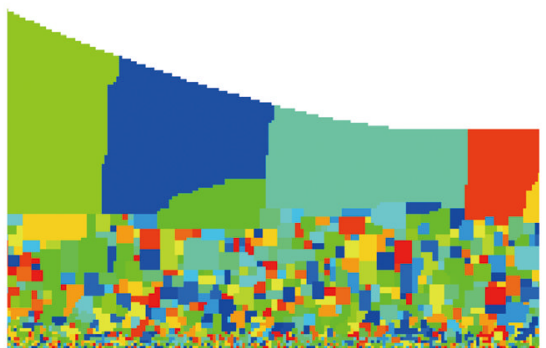

Fig. 8: Illustrations of competitive growth (a1), (b1) and (c1) and grain elimination (a2), (b2) and (c2)

Competitive growth and grain elimination lead to the decrease of available epitaxial growth sites and the increase of grain width. Owing that there is no nucleation happening for the AM of Ti-6-4, controlling the competitive growth of the grain width can be achieved by controlling the orientation of the substrate. The range of initial grain orientation decreases, from $\pm \pi / 4$ to $\pm \pi / 8, \pm \pi / 16$, and 0 . For the convenience of expression, the cases when laser power is $3,000 \mathrm{~W}$, the initial orientation $( \pm \pi / 4, \pm \pi / 8, \pm \pi / 16$ and 0$)$ is denoted as the $3,000 \mathrm{~W}-\pi / 4,3,000 \mathrm{~W}-\pi / 8,3,000 \mathrm{~W}-\pi / 16$, and $3,000 \mathrm{~W}-0$ cases. The average grain width as a function of the deposition layer is shown in Fig. 9.

For the $3,000 \mathrm{~W}-\pi / 4$ case, the change of grain width can be roughly divided into three stages. At Stage A (to the left of the black dotted line) in Fig. 9, the grain width increases sharply, which means the competitive growth is relatively violent. At Stage B (between the black dotted and dashed lines), the grain width increases continually while its first-order derivative is smaller than that in Stage A. Competitive growth with a relatively moderate rate still exists. At Stage $\mathrm{C}$, the surviving 


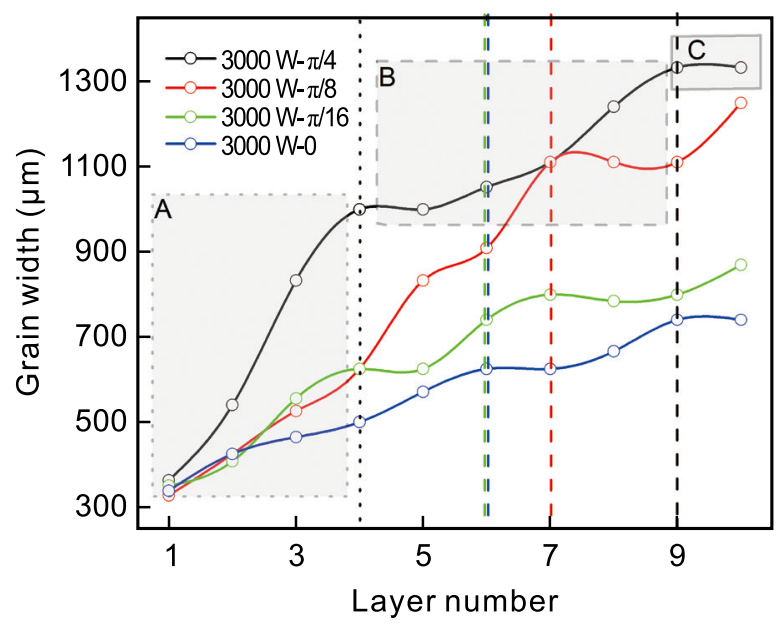

Fig. 9: Illustration of grain width as a function of layer number

grains have almost similar orientations and it is not easy to win or lose its competitive growth. The grain width becomes relatively stable. For the other cases, a similar phenomenon can be found, except that Stage A and Stage B are difficult to distinguish, and in Fig. 9, the dashed lines between Stage B and Stage $\mathrm{C}$ are shown.

The orientation distribution at different deposition layers is shown in Fig. 10. After the deposition of the 1st layer [Fig. 10(a)], the orientation of the surviving grains is among a wide range. After the 5th layer is deposited [Fig. 10(b)], the orientation of the surviving grains is mainly between $-5^{\circ}$ and $10^{\circ}$, although some grains have an orientation less than $-10^{\circ}$. After the 10th layer deposition [Fig. 10(c)], the orientation of the surviving grains is between $-5^{\circ}$ and $10^{\circ}$, but mainly between $-5^{\circ}$ and $5^{\circ}$. The grain orientation after the 10th layer deposition is similar to that after the 5 th layer deposition and far from that after the 1st layer deposition. Therefore, during the stable Stage C, not only does the grain width becomes relatively stable, but also the orientation distribution does not change too much.

Generally, narrowing the grain distribution leads to the decrease of the grain width, which is attributed to the decrease of the grain competitive growth. After the 9th, 7th, 6th, and 6 th layer deposition, the grain width becomes stable for the $3,000 \mathrm{~W}-\pi / 4,3,000 \mathrm{~W}-\pi / 8,3,000 \mathrm{~W}-\pi / 16$, and $3,000 \mathrm{~W}-0$ cases, respectively. It can be found that as the initial orientation distribution becomes narrower, the stable grain width stage will come earlier. An interesting thing is that the layer number to reach a stable grain width stage for the $3,000 \mathrm{~W}-\pi / 16$ and $3,000 \mathrm{~W}-0$ is the same, and the grain width for the two cases is similar during the whole process. According to the distribution of the grain orientation after the 10th layer deposition [Fig. 10(c)], the surviving grains are with the orientation between $-5^{\circ}$ and $10^{\circ}$. In the $3,000 \mathrm{~W}-\pi / 16$, and $3,000 \mathrm{~W}-0$ case, the grains are initialized with the orientation close to $-5^{\circ}$ and $10^{\circ}$. Therefore, the blocked grains during competitive growth are at a relatively small amount. The grain width as a function of deposition layers is similar for the two cases.

The stable stage is of potential importance for additive manufacturing modeling. It is well known that epitaxial growth exists in the AM. In this regard, to simulate the grain structure accurately, it is needed to calculate from the very first layer deposition, which lays a heavy computational cost for the layer deposition far away from the first layer deposition. However, the stable grain width stage indicates there is a possibility that the grain width will become stable when the stage is reached. If we want to investigate the grain evolution at some position far from the first layer deposition, there is no need to calculate it from the first layer deposition anymore and the simulation box is initialized with the stable stage instead.
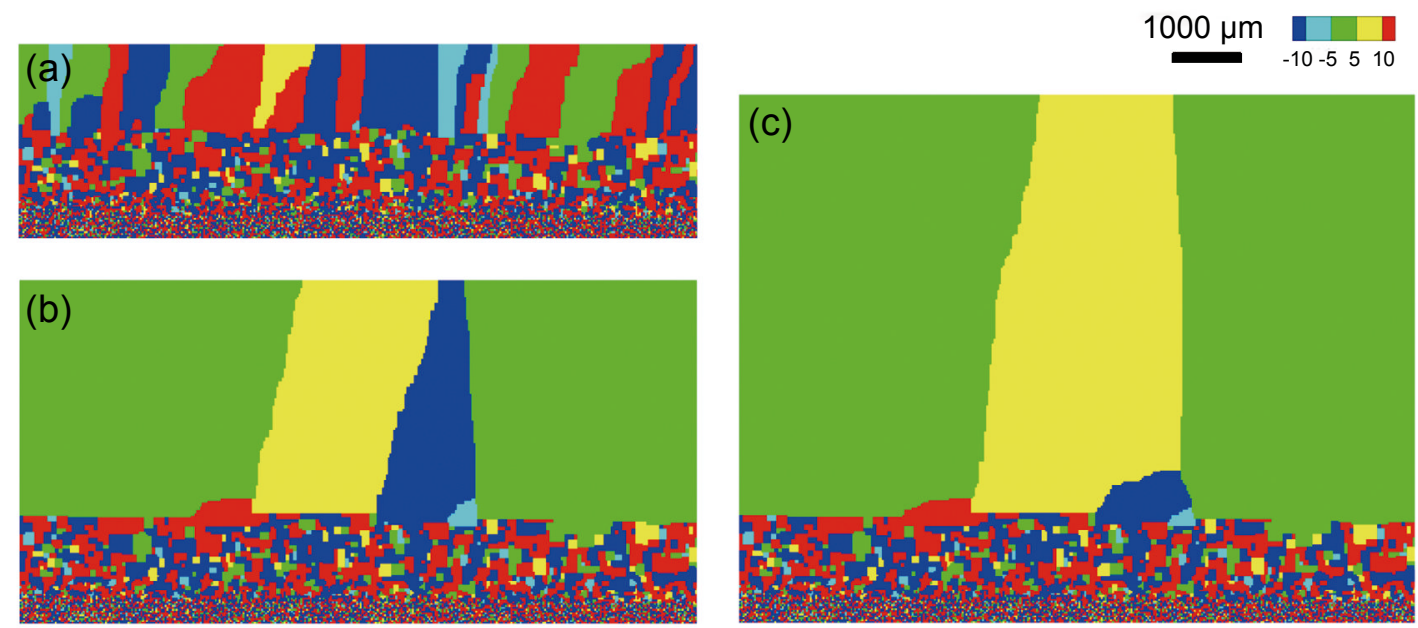

Fig. 10: Illustrations of grain orientation after the 1st (a), 5th (b) and 10th (c) layer deposition when laser power is $3,000 \mathrm{~W}$ and initial orientation distribution is between $-45^{\circ}$ and $45^{\circ}$

\subsection{Molten pool morphology}

The molten pool morphology is important for grain evolution. In the previous section, it has been proved that after competitive growth and grain elimination, the grain with the orientation within some range will survive at last. To avoid the effects of the orientation on the molten pool morphology and considering experimental results ${ }^{[5]}$ show that the grains with the $<001>$ along the building has the potential to survive, the 
cases carried out in this section and the following section are initialized with the orientation of $0^{\circ}$ without further notice.

When the molten pool morphology does not change with time for some particular layer deposition, the grain growth orientation is normal to the tail of the molten pool. In classical welding theory, it is believed that the molten pool is determined by the liquidus, and therefore the growth orientation is along the temperature gradient. Figure 11(a) shows the molten pool determined by the liquidus. It can be found that the molten pool is relatively curved. If the growth orientation is along the temperature gradient, the relatively curved molten pool will lead to relatively curved grains, which violates what has been observed in the experiments (Figs. 6 and 7). On the other hand, the molten pool determined by the $\mathrm{S}-\mathrm{L}$ interface calculated from the CA method exhibits different features, as shown in Fig. 11(b). It can be found that a long lagged and gentle tail exists in Fig. 11(b), which supports the conclusion that the grain with the orientation $<001>$ along the building direction survives.

From Fig. 11(b), it can be found that as the layer is deposited, the length and depth of the rear part of the molten pool increase. The width and depth as a function of layer number are shown in Fig. 12(a). The length and depth are $4.231 \mathrm{~mm}$ and $1.181 \mathrm{~mm}$ after the 1st layer deposited. They are $13.214 \mathrm{~mm}$ and $3.352 \mathrm{~mm}$ after the 10th layer deposited, which are around 3 times of that after the 1st layer deposited. The length and depth of the tail increase as the layer number increases, which lays a heavy burden on the stability of the molten pool. The shape of the molten pool is mainly converged by the surface tension and diverged by its gravity. When the size of the molten pool is relatively large, the liquid will lose its stability and spread because the surface tension is less than its gravity. From Figs. 11(a) and (b), it can be found that the size of molten pool calculated by CA is almost twice the size as from the liquidus. Therefore, when using the liquid temperature to infer the molten pool, its size will be underestimated and its stability will be overestimated.

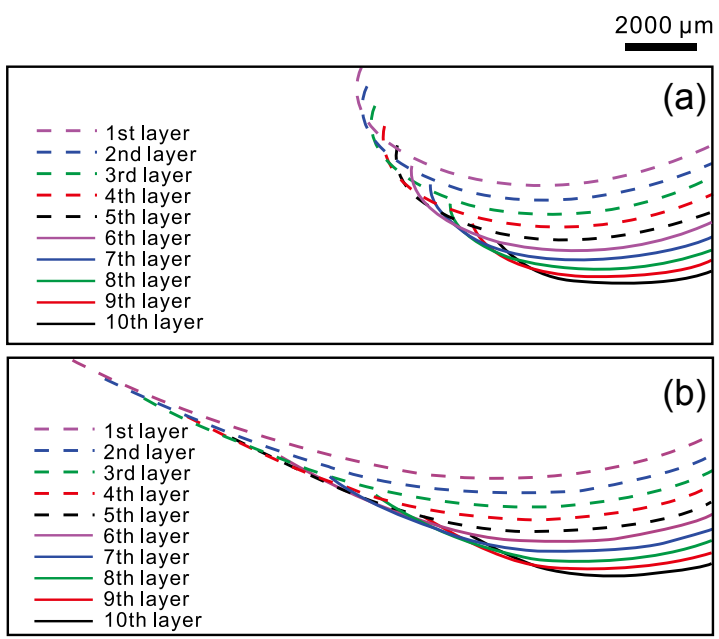

Fig. 11: Molten pool determined by S-L interface calculated from CA (a) and liquidus (b) for ten deposited layers. Scanning and building direction are denoted as $x$ and $y$-direction
The temperature on the S-L interface as a function of the scanning direction is shown in Fig. 12(b). For every deposited layer, the temperature is not isothermal and decreases as the distance grows from the bottom of the molten pool. Low temperature, i.e., large undercooling, means great growth velocity. The growth velocity increases from the bottom of the rear molten pool to the top. As the deposited layer increases, the temperature at the same position increases as well. The grain will grow slower at the same position for the later layer deposition. It can also be found that the slope of the temperature with respect to building direction $X$, which is defined as the distance from the laser center, decreases as the layer number increases. It is mainly attributed to the low heat transfer coefficient of Ti-6-4. When there is accumulated and redundant heat, the grains will grow slower and thus the molten pool will become larger. If the minimum temperature for different layers is connected and shown as a function of $X$ [the orange dot line in Fig. 12(b)], it is reasonable to anticipate that the minimum temperature decreases and the length of the tail increases when there are more deposited layers, which further lays a heavy burden on the stability of the molten pool.

A well-accepted method to stabilize the molten pool is to decrease the line energy density, which can be realized by either increasing the scanning velocity or decreasing the laser power. Here, the effects of decreasing the laser power were investigated. The depth and length of the rear part of the molten pool [Fig. 12(a)] increase continually when the layer number increases. After the 1st layer deposited, the depth and length are $3.170 \mathrm{~mm}$ and $0.690 \mathrm{~mm}, 3.681 \mathrm{~mm}$ and $0.989 \mathrm{~mm}$, and $4.231 \mathrm{~mm}$ and $1.181 \mathrm{~mm}$ when the power is $2,500 \mathrm{~W}, 2,750 \mathrm{~W}$ and 3,000 W, respectively. After the 10th layer deposited, the depth and length are $9.397 \mathrm{~mm}$ and $2.349 \mathrm{~mm}, 11.319 \mathrm{~mm}$, and $2.939 \mathrm{~mm}$, and $13.214 \mathrm{~mm}$ and $3.352 \mathrm{~mm}$ when the power is $2,500 \mathrm{~W}, 2,750 \mathrm{~W}$ and $3,000 \mathrm{~W}$, respectively. It can be found the depth and width increase twice. As the layer number increases further, it is reasonable to anticipate that the depth and length increase further, which still imposes a burden on the stability of the molten pool. Therefore, decreasing laser power seems not to help in increasing the stability of the molten pool too much for this case.

The temperature on the S-L interface as a function of $X$ is also analyzed when the laser power is $2,750 \mathrm{~W}$ [Fig. 12(c)] and 2,500 W [Fig. 12(d)]. The temperature on the S-L interface also exhibits the same features as it obtained from the 3,000 W case. An interesting thing is for the initial deposited layers, the difference of the minimum temperatures among the three cases is clear. For example, after the 1st layer deposited, the minimum temperature is $1,813.78 \mathrm{~K}, 1,792.80 \mathrm{~K}$, and $1,761.16 \mathrm{~K}$ for the $2,500 \mathrm{~W}, 2,750 \mathrm{~W}$, and $3,000 \mathrm{~W}$ cases. After the 7th layer deposited, the minimum temperature is $1,541.09 \mathrm{~K}, 1,528.24 \mathrm{~K}$, and $1,522.05 \mathrm{~K}$ for the $2,500 \mathrm{~W}, 2,750 \mathrm{~W}$, and 3,000 $\mathrm{W}$ cases, respectively. As the deposited layer number increases, the difference gradually decreases, and even disappears, and finally, the minimum temperature is very similar and seems to be independent of the laser power density. The low heat transfer 

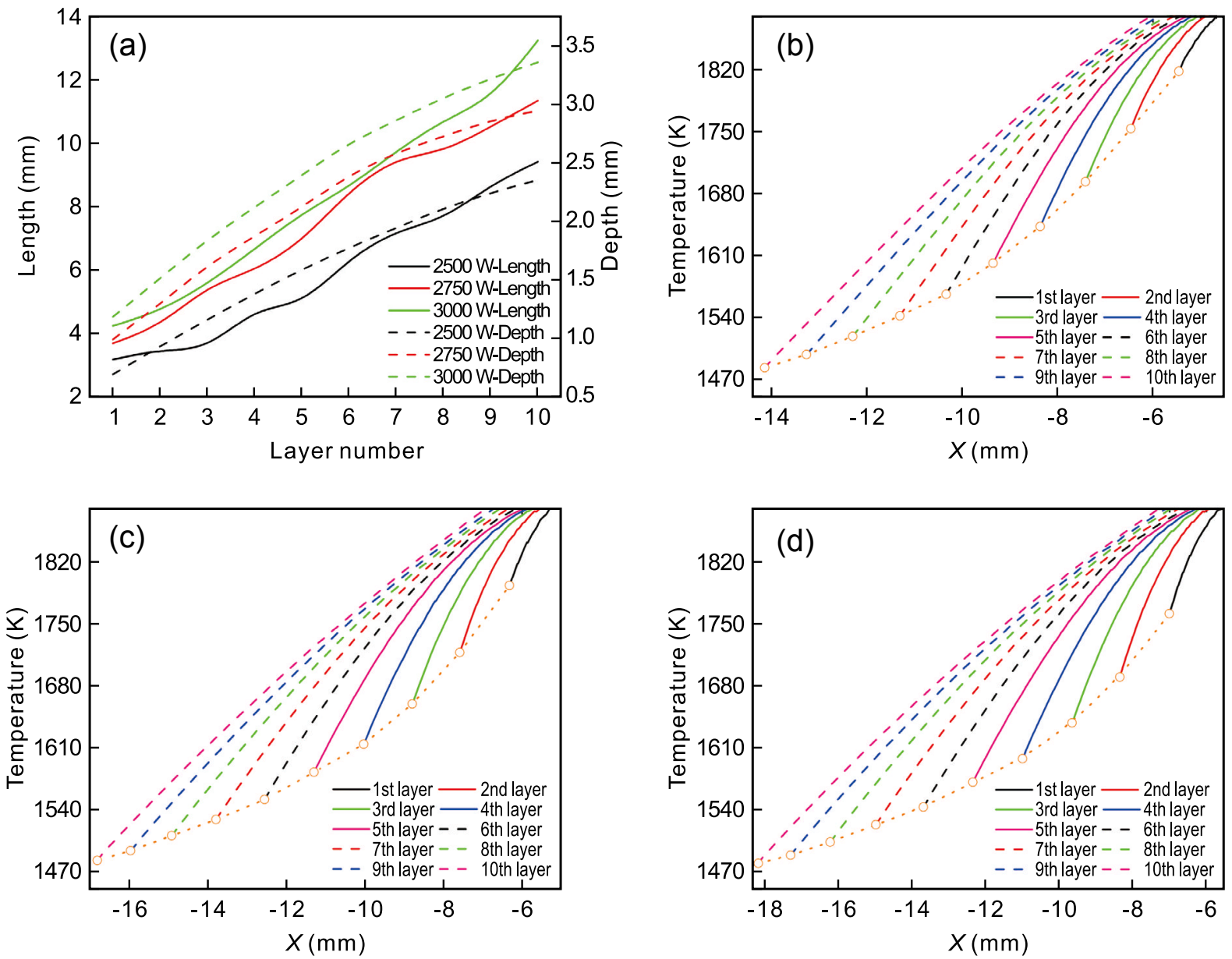

Fig. 12: Length and depth of rear part of molten pool (a), and temperature at S-L interface as a function of building direction $(X)$ under different laser powers: (b) 3,000 W; (c) 2,750 W; (d) 2,500 W (origin of building direction is position of laser power)

coefficient leads to too much heat on the component and hereby the grain grows slowly. Then the molten pool has to be extended to the relatively low temperature. At the moment, it is the temperature distribution that dominates the grain growth. To increase the stability of the molten pool, decreasing line power density works at the beginning, but shows few effects when there is too much redundant heat in the substrate and component.

\subsection{Grain growth orientation}

The grain morphology obtained from the simulation is affected by both the grain growth in the molten pool and the grain coarsening in the HAZ. To eliminate the effects of the latter, the grain growth path is calculated according to the molten pool morphology.

Suppose the morphology of the molten pool does not change during the depositing for each layer. The assumption stands when the temperature distribution becomes relatively stable. Except for the initial and the final stage in which the thermal distribution is affected by the edge, the temperature distribution is stable for the most time. In this regard, the grain growth orientation is normal to the tail of the molten pool, and thus the growth orientation can be deduced from the molten pool morphology. As shown in Fig. 13(a), for every point at the S-L interface, the angle between its normal and the building direction (denoted as the $y$-direction) is denoted as $\alpha$. When the laser moves from left to right (denoted as the positive $x$-direction), the grain experiences the heat history as the temperature from the bottom to the top of the rear part of the molten pool. Suppose the path from the bottom to the top of the rear part of the molten pool is $\vec{l}$, when the position of the $\mathrm{S}-\mathrm{L}$ interface of specific grain is $p$ (a point belonging to the path $\vec{l}$ ), the relation between the grain growth in $x$-direction and $y$-direction is:

$$
d x=\tan (p(\alpha)) d y
$$

where $d x$ and $d y$ are the infinitesimals of the grain growth in $x$-direction and $y$-direction. Integrate Eq. (4) with the path $\vec{l}$, i.e:

$$
x=\int_{\vec{i}} \tan (p(\alpha)) d y
$$

Then, the growth direction is defined as the inclined angle $\beta$ from the building direction, which is:

$$
\beta=\arctan \frac{x}{y}
$$

Figure 13(b) shows the illustration of $\alpha$ as a function of $y$ when the laser power is $3,000 \mathrm{~W}$. Since $\alpha$ is the growth direction, the grain will have more potential to survive if 
(a)
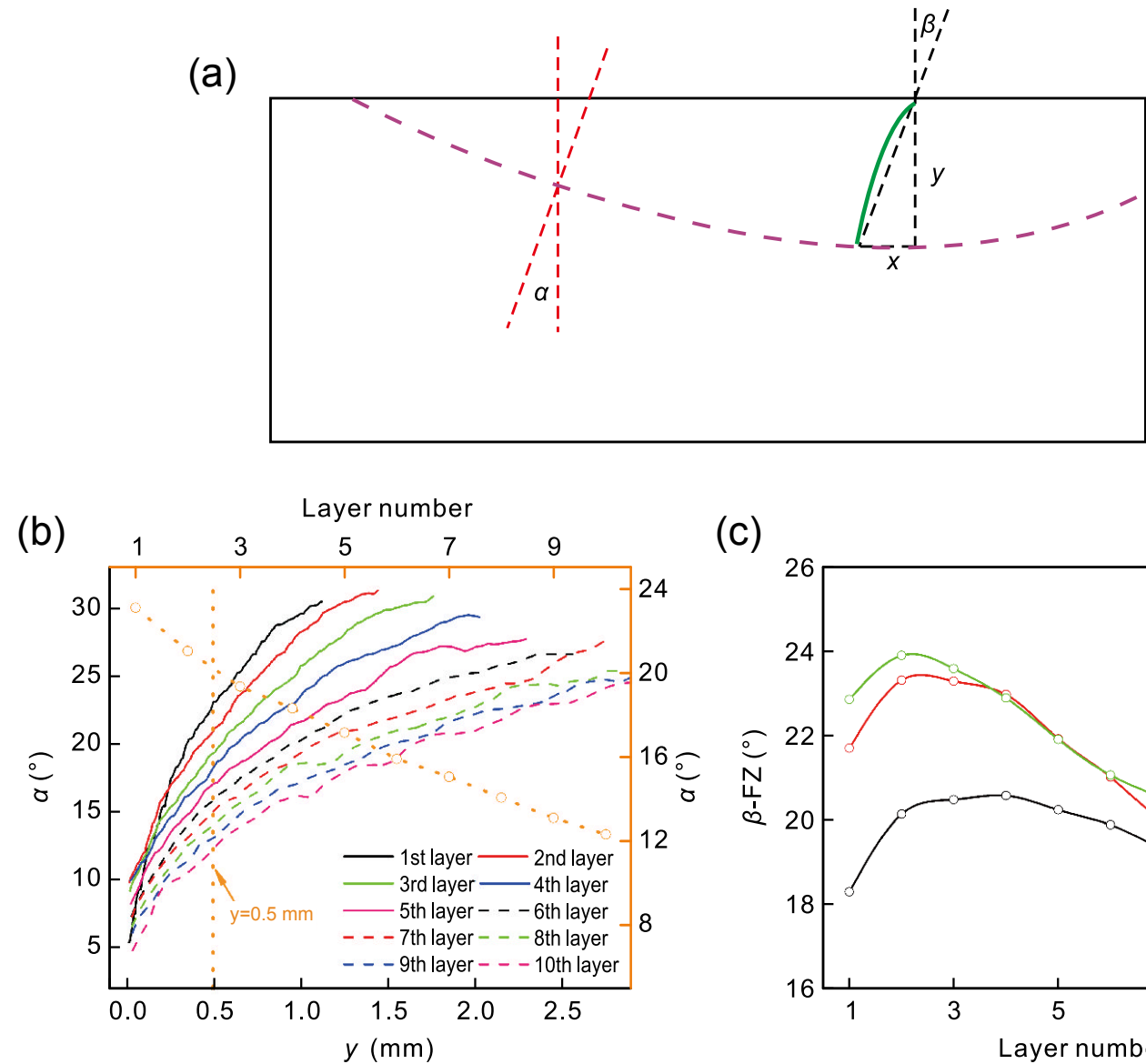

(c)

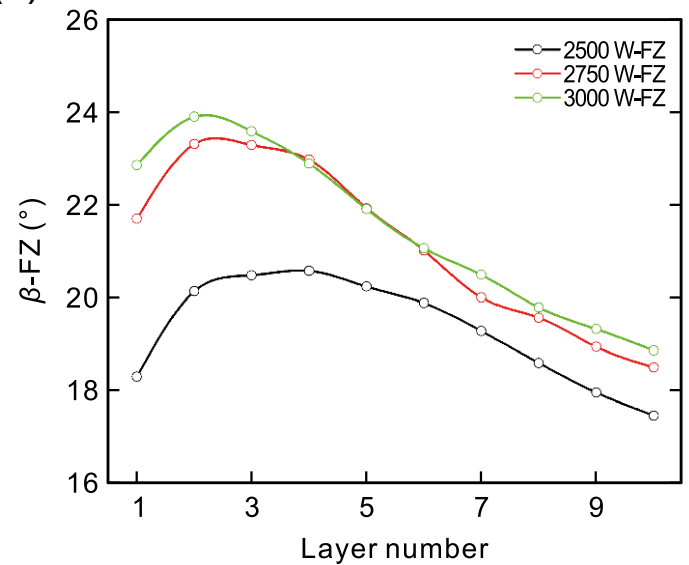

Fig. 13: Schematic of relation between molten pool (pink dashed line) and grain morphology (green solid line) (a); $\alpha$ is angle between normal of any point at molten pool and building direction $(y) . \beta$ is angle inclined from building direction. When laser power is $3,000 \mathrm{~W}, \alpha$ as a function of $y$, in which $y$ is aligned by bottom of molten pool (b); Illustrations of $\beta$ as a function of layer number (c)

its orientation is closer to $\alpha$. However, the problem is the favorable orientation varies with the position of $y$. The difference between the bottom and the top of the molten pool morphology cannot be ignored. Take the tenth deposition as an example, at the bottom of the molten pool, $\alpha$ is $5.840^{\circ}$, while it is $24.779^{\circ}$ at the top of the molten pool. Therefore, the conclusion cannot be drawn directly that the grain with the orientation along the building direction will survive.

An interesting thing is that the $\alpha$ value tends to decrease with the increase of layer number. If extracting the $\alpha$ value at $y=0.5 \mathrm{~mm}$, it can be found that the value decreases steadily monotonously [the dotted orange line in Fig. 13(b)]. Therefore, with the layer-and-layer deposition, the grain with the $<01>$ direction close to the building direction has greater and greater potential to survive. Considering CET is not likely to happen during the AM of Ti-6-4, the available nucleation sites are from the substrate or the previously deposited layer. In Section 3.2, it is proved that competitive growth and grain elimination leading to the orientation of the final surviving grains after layer-and-layer grain selection is concentrated to a specific range. To sum up, the flat rear part of molten pool makes the grain with favorable orientation have more potential to survive. The mechanism of grain competitive growth and elimination removes the grain with unfavorable orientation.
Further, extending the $2 \mathrm{D}$ conclusion to the $3 \mathrm{D}$, which is with the increase of the layer number, grains with the $<001>$ direction close to the building direction has more potential to survive. After layer-and-layer deposition, it is reasonable to anticipate the grain with the orientation along the building direction will survive at last.

The growth orientation (the inclined angle $\beta$ ) of the grains in the FZ is analyzed and shown in Fig. 13(c). For the three cases, the inclined angle increases firstly and then decreases with the layer number, which is consistent with what was observed in experiments ${ }^{[26]}$. The maximum inclined angle value is obtained when the layer number is 2 or 3 . When the laser power increases, the inclined angle increases as well. However, when the deposition number increases to a certain level, the effects of the laser power on the inclined angle are negligible. As it can be found in Fig. 13(c), after the 1st layer deposition, the difference of $\beta$ value between the $3,000 \mathrm{~W}$ and $2,500 \mathrm{~W}$ case is $4.572^{\circ}$, while it is $1.403^{\circ}$ after the 10 th layer deposition.

\section{Conclusions}

In this research, the grain selection and growth orientation of prior- $\beta$ phase for Ti-6-4 during AM are investigated by the 
multi-scale simulation. Main conclusions and highlights of the research are:

(1) The accuracy of the simulation of the grain evolution of Ti-6-4 during AM using a multiscale microstructure simulation framework is validated by experiments in both qualitative and quantitative ways.

(2) The competitive growth and grain elimination are investigated by in-situ grain evolution. Grains with unfavorable orientation lose the competitive growth with their neighbors and then there will be a great chance that the blocked grain will be eliminated, driven by the decrease of the grain boundary.

(3) The molten pool morphology is obtained by the multiscale simulation. The size of the molten pool increases remarkably as the layer number increases, which lays a heavy burden on the stability of the molten pool. Decreasing the line power energy is helpful at the beginning but shows fewer and fewer effects as the deposited layer increases.

(4) The analytical relationship between the grain growth orientation and the molten pool morphology is deduced. With the increase of the layer number, grains with the $<001>$ direction close to the building direction have greater potential to survive.

\section{Acknowledgements}

This research was supported by the National Key Research and Development Program of China (No. 2017YFB1103700) and the National Natural Science Foundation of China (No. 51575304 and No. 51674153).

\section{References}

[1] Kobryn P A, Semiatin S. Microstructure and texture evolution during solidification processing of Ti-6Al-4V. Journal of Materials Processing Technology, 2003, 135(2-3): 330-339.

[2] Ren $Y M$, Lin $X, F u X$, et al. Microstructure and deformation behavior of Ti-6Al-4V alloy by high-power laser solid forming. Acta Materialia, 2017, 132: 82-95.

[3] Carroll B E, Palmer T A, Beese A M. Anisotropic tensile behavior of Ti-6Al-4V components fabricated with directed energy deposition additive manufacturing. Acta Materialia, 2015, 87: 309-320.

[4] Thijs L, Verhaeghe F, Craeghs T, et al. A study of the microstructural evolution during selective laser melting of Ti6Al-4V. Acta Materialia, 2010, 58(9): 3303-3312.

[5] Antonysamy A A, Meyer J, Prangnell P. Effect of build geometry on the $\beta$-grain structure and texture in additive manufacture of Ti-6Al-4V by selective electron beam melting. Materials Characterization, 2013, 84: 153-168.

[6] Li J J, Wang Z J, Wang Y Q, et al. Phase-field study of competitive dendritic growth of converging grains during directional solidification. Acta Materialia, 2012, 60(4): 14781493.

[7] Hu M D, Sun C, Fang $H$, et al. Competitive dendrite growth during directional solidification of a transparent alloy: Modeling and experiment. The European Physical Journal E, 2020, 43(3): 1-11.
[8] Deschamps J, Georgelin M, Pocheau A. Growth directions of microstructures in directional solidification of crystalline materials. Physical Review E, 2008, 78(1): 011605.

[9] Chen S J, Guillemot G, Gandin C-A. Three-dimensional cellular automaton-finite element modeling of solidification grain structures for arc-welding processes. Acta Materialia, 2016, 115: 448-467.

[10] DebRoy T, Wei H L, Zuback J S, et al. Additive manufacturing of metallic components-Process, structure and properties. Progress in Materials Science, 2018, 92: 112-224.

[11] Sun W Z, Yan R, Zhang Y Z, et al. GPU-accelerated threedimensional large-scale simulation of dendrite growth for Ti6Al4V alloy based on multi-component phase-field model. Computational Materials Science, 2019, 160: 149-158.

[12] Zinovieva O, Zinoviev A, Ploshikhin V. Three-dimensional modeling of the microstructure evolution during metal additive manufacturing. Computational Materials Science, 2018, 141: 207-220.

[13] Zhu M F, Tang $Q Y$, Zhang Q Y, et al. Cellular automaton modeling of micro-structure evolution during alloy solidification. Acta Metallurgica Sinica, 2016, 52(10): 1297-1310.

[14] Sun W Z, Xie Y, Yan R, et al. A new efficient quantitative multicomponent phase field: Lattice Boltzmann model for simulating Ti6AI4V solidified dendrite under forced flow. Metallurgical and Materials Transactions B, 2019.

[15] Gandin C A, Carozzani T, Digonnet H, et al. Direct modeling of structures and segregations up to industrial casting scales. JOM, 2013, 65(9): 1122-1130.

[16] Gandin C A, Rappaz M. A coupled finite element-cellular automaton model for the prediction of dendritic grain structures in solidification processes. Acta Metallurgica et Materialia, 1994, 42(7): 2233-2246.

[17] Koepf J A, Gotterbarm M R, Markl M, et al. 3D multi-layer grain structure simulation of powder bed fusion additive manufacturing. Acta Materialia, 2018, 152: 119-126.

[18] Li X X, Tan W D. Numerical investigation of effects of nucleation mechanisms on grain structure in metal additive manufacturing Computational Materials Science, 2018, 153: 159-169.

[19] Wei H L, Elmer J W, DebRoy T. Three-dimensional modeling of grain structure evolution during welding of an aluminum alloy. Acta Materialia, 2017, 126B: 413-425.

[20] Rappaz M, Gandin C A. Probabilistic modelling of microstructure formation in solidification processes. Acta Metallurgica et Materialia, 1993, 41(2): 345-360.

[21] Gandin C A, Rappaz M. A 3D cellular automaton algorithm for the prediction of dendritic grain growth. Acta Materialia, 1997, 45(5): 2187-2195.

[22] Rai A, Helmer H, Körner C. Simulation of grain structure evolution during powder bed based additive manufacturing. Additive Manufacturing, 2017, 13: 124-134.

[23] Rodgers T M, Madison J D, Tikare V. Simulation of metal additive manufacturing microstructures using kinetic Monte Carlo. Computational Materials Science, 2017, 135: 78-89.

[24] Goldak J, Chakravarti A, Bibby M. A new finite element model for welding heat sources. Metallurgical Transactions B, 1984, 15(2): 299-305.

[25] Hu R Z, Chen X, Yang G, et al. Metal transfer in wire feedingbased electron beam 3D printing: Modes, dynamics, and transition criterion. International Journal of Heat and Mass Transfer, 2018, 126: 877-887.

[26] Yin J, Peng G Y, Chen C P, et al. Thermal behavior and grain growth orientation during selective laser melting of Ti-6Al-4V alloy. Journal of Materials Processing Technology, 2018, 260: $57-65$. 